\title{
1 Density-functional and tight-binding theory of silicene and silicane
}

\author{
V. Zólyomi ${ }^{1}$, N. D. Drummond ${ }^{2}$, J. R. Wallbank ${ }^{1}$, and V. I. Fal'ko ${ }^{1}$ \\ ${ }^{1}$ National Graphene Institute, University of Manchester, Booth Street East, \\ Manchester M13 9PL, United Kingdom ${ }^{2}$ Physics Department, Lancaster \\ University, Lancaster, LA1 4YB, United Kingdom
}

\begin{abstract}
Summary. A combination of density functional theory and a tight-binding model offers a robust means to describe the structure, vibrations, and electronic states of silicene. In this chapter we give an overview of the electronic structure and phonon dispersions of silicene and its fully hydrogenated derivative, silicane. We discuss the dynamical stability of the buckled silicene and silicane lattices and we present their phonon dispersions. We discuss the first-principles electronic band structure of ideal, free-standing silicene, paying particular attention to the small band gap opened by spin-orbit coupling, which renders the material a topological insulator. We look at the tight-binding description of silicene and examine the effects of an external electric field which, above a critical electric field, counters the spin-orbit gap and triggers a phase transition into a band-insulator state in which the band gap is linearly tunable by the electric field. We also present the tight-binding description of silicane which, parameterised by density functional theory, sheds light on the importance of long-range hopping in this material.
\end{abstract}

\subsection{Introduction}

Two-dimensional (2D) crystals exist in a broad variety of forms, perhaps the simplest of which is graphene $[1,2]$. Forming an atomically thin honeycomb lattice with two atoms in its unit cell, it is a high-symmetry $2 \mathrm{D}$ crystal with versatile physical properties. The next simplest $2 \mathrm{D}$ crystal comes to us in the form of the silicon equivalent of graphene: silicene $[3,4]$. The difference in structure between graphene and silicene takes the form of a sublattice buckling, illustrated in Fig. 1.1. The $A$ and $B$ sublattices of the crystal do not lie in the same plane. For this reason, silicene has reduced symmetry in comparison to graphene, which has important consequences for its physical properties.

Silicene does not occur in nature, but monolayers have been synthesised on $\operatorname{Ag}(111)$ surfaces [5-13]. Due to the similarity of the lattice structures, the band structure of silicene resembles that of graphene, featuring Dirac-type electron dispersion in the vicinity of the corners of its hexagonal Brillouin zone (BZ) [14]. Moreover, silicene has been shown theoretically to be metastable as a free-standing $2 \mathrm{D}$ crystal $[3,4]$, implying that it is possible to transfer silicene 
a)
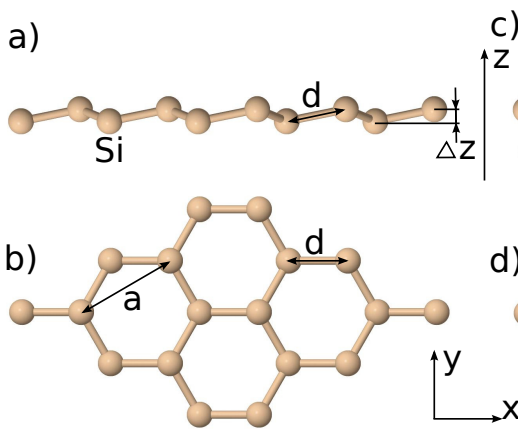

c) $\quad W$

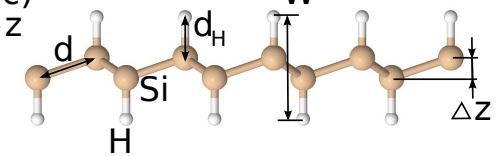

$\mathrm{H}$

d)

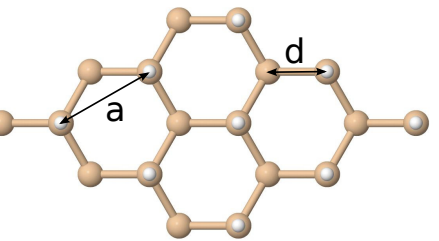

Fig. 1.1. Atomic structure of silicene (a: side view, b: top view) and silicane (c: side view, $\mathrm{d}$ : top view). The key structural parameters are marked in the figure: $a$ is the lattice parameter, $d$ is the $\mathrm{Si}-\mathrm{Si}$ bond length, $\Delta z$ is the sublattice buckling, $d_{\mathrm{H}}$ is the $\mathrm{Si}-\mathrm{H}$ bond length, and $W$ is the width of the silicane sheet. The structural parameters are summarised in Table 1.1

Table 1.1. Structural parameters of silicene and silicane (see Fig. 1.1) in $\AA$ units according to density functional theory are summarised below. The local density approximation (LDA) and Perdew-Burke-Ernzerhof (PBE) exchange-correlation functionals are used

\begin{tabular}{lcccccc}
\hline Crystal & Method & $a$ & $d$ & $\Delta z$ & $d_{\mathrm{H}}$ & $W$ \\
\hline Silicene & LDA & 3.83 & 2.21 & 0.44 & & \\
Silicene & PBE & 3.87 & 2.23 & 0.45 & & \\
Silicane & LDA & 3.78 & 2.31 & 0.74 & 1.51 & 3.76 \\
Silicane & PBE & 3.84 & 2.34 & 0.74 & 1.51 & 3.76 \\
\hline
\end{tabular}

onto an insulating substrate and gate it electrically. Recently, a silicene fieldeffect transistor was fabricated by capping the silicene with $\mathrm{Al}_{2} \mathrm{O}_{3}$ before transferring it off the $\mathrm{Ag}(111)$ surface on which it was grown [13].

The similarity between graphene and silicene arises from the fact that $\mathrm{C}$ and $\mathrm{Si}$ belong to the same group in the periodic table of elements. However, Si has a larger ionic radius, which promotes $\mathrm{sp}^{3}$ hybridisation, whereas $\mathrm{sp}^{2}$ hybridisation is energetically more favourable in $\mathrm{C}$. As a result, in a $2 \mathrm{D}$ layer of $\mathrm{Si}$ atoms, the bonding is formed by mixed $\mathrm{sp}^{2}$ and $\mathrm{sp}^{3}$ hybridisation. Hence silicene is slightly buckled, with one of the two sublattices of the honeycomb lattice being displaced vertically with respect to the other, as shown in Fig. 1.1. Such buckling creates new possibilities for manipulating the dispersion of electrons in silicene and opening an electrically controlled sublattice-asymmetry band gap [15]. In fact, by applying an external electric field to silicene it becomes a semiconductor with a tunable band gap $\Delta$ that can reach tens of meV before the $2 \mathrm{D}$ crystal loses structural stability [4]. But even at zero electric field, silicene is a gapped material, due to 
the Kane-Mele spin-orbit (SO) coupling [16] for electrons on a honeycomb lattice, which opens a small SO-gap at the Brillouin zone corner and renders the material a topological insulator $[17,18]$.

The ability to open a band gap in silicene highlights one of the limitations in the practical applicability of monolayer graphene, namely that it has no band gap. Due to the lack of a sub-lattice buckling, the SO-gap in graphene is negligible, and no gap appears upon the application of an electric field, either. Hence plenty of research has gone into insulating and semiconducting $2 \mathrm{D}$ crystals, such as boron nitride [19,20], transition metal dichalcogenides [21-31], and III-VI chalcogenides [32-35].

Yet band-gap engineering in graphene has also been shown to be possible by chemical means, as complete hydrogenation turns graphene into a gapped material. The resulting material, graphane [36] $\left(\mathrm{C}_{2} \mathrm{H}_{2}\right)$ has a buckled honeycomb structure with a single hydrogen atom attached to each carbon site on alternating sides of the sheet. Experiment [37] has shown that few-layer germanane $\left(\mathrm{Ge}_{2} \mathrm{H}_{2}\right)$, hydrogenated germanene, can be synthesised, expanding the family of atomic $2 \mathrm{D}$ materials. It is expected that silicane will be stable as well. Density functional theory (DFT) predicts that not only is silicane stable, but it exhibits a sizable indirect band gap and a strongly anisotropic conduction-band edge, which is a consequence of long-range hopping interactions within the crystal [38].

In this chapter we overview the properties of silicene and silicane as revealed by DFT and a tight-binding model, highlighting the tunable band gap of silicene in an external electric field.

\subsection{First-principles theory of silicene and silicane}

\subsubsection{Structure, stability, and electronic band structure of silicene}

In the optimal structure of silicene the $z$-coordinates of the two Si atoms in the unit cell (the $A$ and $B$ sublattices) differ by a finite distance $\Delta z$, shown in Fig. 1.1 and Table 1.1. This metastable lattice is the same as the "low-buckled" structure found by Cahangirov et al. [3]. The parameters were obtained as follows.

First-principles DFT was employed to calculate the optimal structure of freestanding silicene, using the CASTEP $[39,40]$ and VASP [41] plane-wave-basis codes, employing ultrasoft pseudopotentials and the projector-augmentedwave (PAW) method, respectively. For the exchange-correlation functional the local density approximation (LDA), the Perdew-Burke-Ernzerhof (PBE) generalised gradient approximation [42], and the screened Heyd-ScuseriaErnzerhof 06 (HSE06) hybrid functionals were used [43,44].

All plane-wave DFT total energies were corrected for finite-basis error [45] and it was verified that the residual dependence of the total energy on the plane-wave cutoff energy is negligible. Ultrasoft pseudopotentials were used 
throughout, except where otherwise stated. The silicene system was made artificially periodic in the $z$ direction (normal to the silicene layer). The atomic structure was obtained by relaxing the lattice parameter and atom positions within DFT.

To evaluate the Fermi velocity shown in Table 1.2 the DFT band structure was calculated using a $53 \times 53 \mathrm{k}$-point grid and a plane-wave cutoff energy of $816 \mathrm{eV}$ in a cell of length $L_{z}=26.46 \AA$. Then Eq. (17) of Ref. [46] was fitted to the highest occupied and lowest unoccupied bands within a circular region around the K point; the Fermi velocity is one of the fitting parameters. The radius of the circular region was $6 \%$ of the length of the reciprocal lattice vectors; the Fermi velocity was converged with respect to this radius.

Table 1.2. Silicene structural and electronic parameters: lattice constant $a$, sublattice buckling $\Delta z$ (the difference between the $z$ coordinates of the $A$ and $B$ sublattices), and Fermi velocity $v$.

\begin{tabular}{lccc}
\hline Method & $a(\AA)$ & $\Delta z(\AA)$ & $v\left(10^{5} \mathrm{~ms}^{-1}\right)$ \\
\hline PBE (CASTEP) & 3.86 & 0.45 & 5.27 \\
PBE (VASP) & 3.87 & 0.45 & 5.31 \\
PBE [15] & 3.87 & 0.46 & \\
LDA (CASTEP) & 3.82 & 0.44 & 5.34 \\
LDA (VASP) & 3.83 & 0.44 & 5.38 \\
LDA [3] & 3.83 & 0.44 & $\approx 10$ \\
LDA [47] & 3.86 & 0.44 & \\
HSE06 (VASP) & 3.85 & 0.36 & 6.75 \\
\hline
\end{tabular}

The experimental measurement of the structural properties of silicene is invariably affected by the $\mathrm{Ag}(111)$ substrate on which the silicene is grown. A detailed discussion of theoretically and experimentally determined structural properties of silicene on various substrates can be found in Ref. [48].

By calculating the DFT phonon dispersion it has been verified $[3,4]$ that free-standing monolayer silicene is dynamically stable: no imaginary frequencies appear anywhere in the BZ. The results of such an analysis are summarised in Fig. 1.2. This stability proves that, as a metastable 2D crystal, silicene can be transferred onto an insulating substrate, where its electronic properties can be studied and manipulated.

The geometry optimisation and band-structure calculations were performed with both the CASTEP $[39,40]$ and VASP [41] codes, to verify that the results are in good agreement. The PAW method [49] was used in the VASP calculations, whereas ultrasoft pseudopotentials were used in the CASTEP calculations. As can be seen in Table 1.2, the geometries predicted by the two codes agree well, and it was verified that the phonon dispersions obtained 


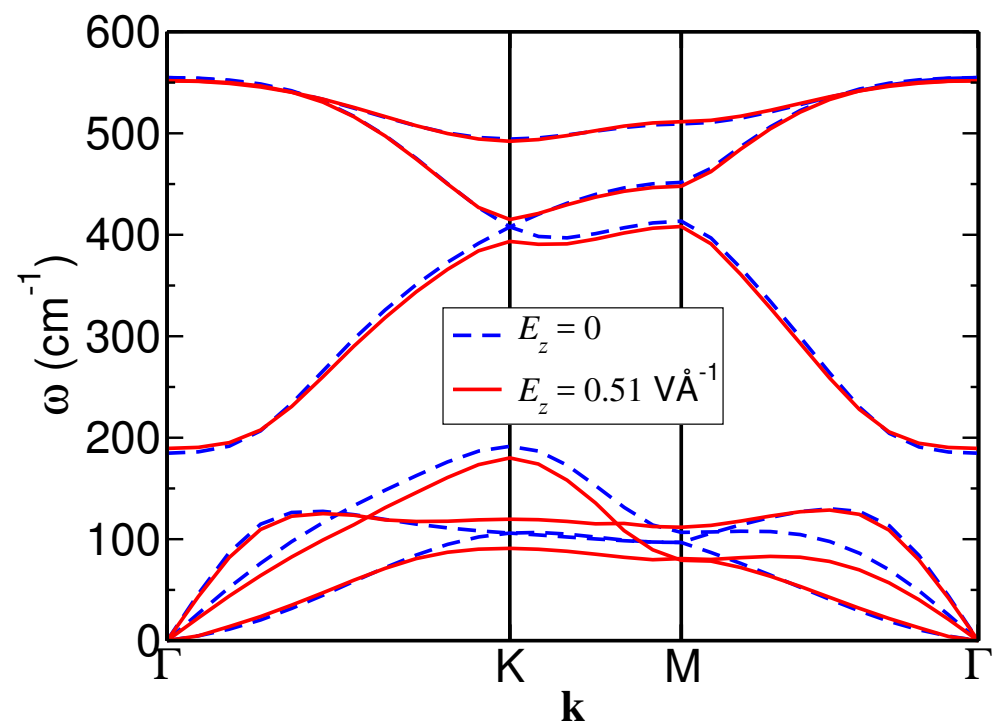

Fig. 1.2. DFT-PBE phonon dispersion curves for silicene in zero external field and at an external transverse electric field of $E_{z}=0.51 \mathrm{~V}^{-1}$. In both cases the calculations were performed using the method of finite displacements, with the atomic displacements being $0.0423 \AA$, in a supercell consisting of $3 \times 3$ primitive cells with a $20 \times 20 \mathbf{k}$-point grid in the primitive cell

with the two codes are virtually identical when the same parameters are used. It was also verified that the phonon dispersion curves are converged with respect to supercell size.

The calculated electronic band structure of a "free" silicene layer is shown in Fig. 1.3. As expected, it resembles the band structure of graphene; in particular it shows the linear Dirac-type dispersion of electrons near the $\mathrm{K}$ points, where the Fermi level in undoped silicene is found.

\subsubsection{Structure, stability, and electronic band structure of silicane}

To obtain the optimal crystal structure, the phonon dispersions, and the electronic band structure of silicane, the VASP [41] plane-wave-basis code was used and the plane-wave cutoff energy was set to $500 \mathrm{eV}$. A $12 \times 12$ Monkhorst-Pack k-point grid was used for geometry optimisations while a $24 \times 24$ grid was used to calculate the band structures. The vertical separation of periodic images of the monolayer was set to $15 \AA$. The force tolerance in the optimisation was $0.005 \mathrm{eV} / \AA$. Phonons were calculated with the forceconstant approach in a $3 \times 3$ supercell. For an estimate of the band gap, the HSE06 exact-exchange functional was used. 


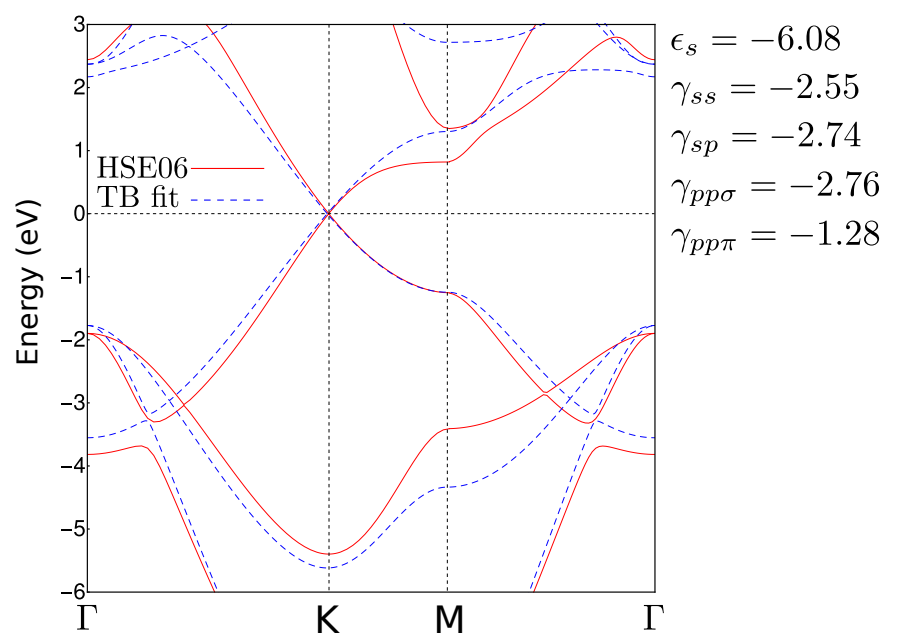

Fig. 1.3. HSE06 band structure of freestanding silicene compared to a tight-binding fit as described in the text. Tight-binding model parameters are listed on the right hand side

The relaxed structure of silicane [38] is very similar to that of graphane, as illustrated in Fig. 1.1. The bond lengths (see Table 1.1) obtained with the PBE functional are systematically larger than those optimised with the LDA, as expected [50]. Note that the hydrogenation is accompanied by a significant increase in the magnitude of the sublattice buckling when compared to silicene. Lattice constants and sublattice bucklings agree with previous literature to within $\pm 5 \%$ and $\pm 10 \%$, respectively [51-55].

The calculated electronic band structures are plotted in Fig. 1.4. One important difference between graphane [56] and silicane is that in the latter material a band appears close to the conduction-band edge at the M point. In fact the conduction-band minimum of silicane is at the $\mathrm{M}$ point, making silicane an indirect-gap semiconductor. The band gap of silicane is $2.91 \mathrm{eV}$ according to the HSE06 functional, which is expected to underestimate the gap by no more than $10 \%$ [57]. Note that the conduction band is anisotropic at the $\mathrm{M}$ point with a heavy effective mass in the $\mathrm{M}-\Gamma$ direction. The finding that the band gap of silicane is indirect is supported by a variety of methods ranging from semilocal DFT through hybrid functionals to single-shot $G W$ $[51,52,54,55]$.

Now we discuss the orbital composition of the valence and conduction bands of silicane (see Table 1.3). At the $\Gamma$ point the valence band consists of Si $p_{x}$ and $p_{y}$ orbitals, while the conduction band is predominantly $\mathrm{Si} s$ and $p_{z}$. However, at the $\mathrm{M}$ point the conduction band also contains Si $p_{x}$ and $p_{y}$ contributions. The $\mathrm{H} s$ orbital also contributes to the valence band in silicane at the $\mathrm{M}$ point. This means that for a tight-binding description of silicane an 


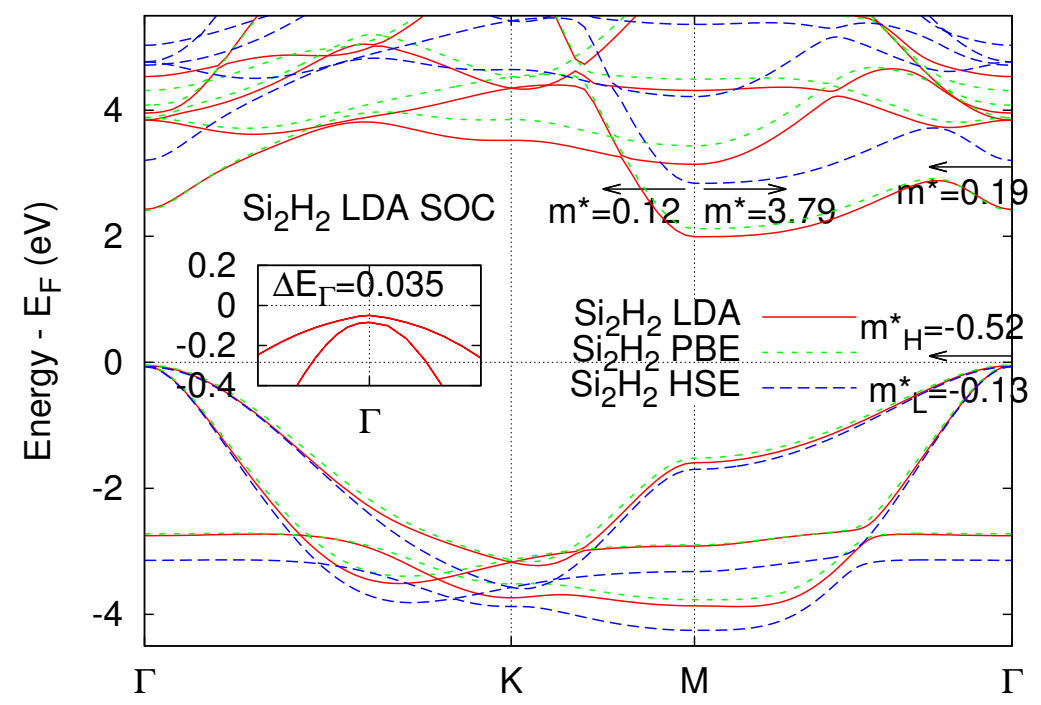

Fig. 1.4. Band structure of silicane. The zero of energy is taken to be the Fermi level and the top of the valence band is marked with a horizontal line. The effect of SO coupling at the $\Gamma$ point is illustrated in the inset. Effective masses (in units of electron mass) in the HSE06 calculations are provided in the conduction band at $\mathrm{M}$ and $\Gamma$, and in the valence band at $\Gamma$ (where the $H$ and $L$ subscript refers to the heavy and light effective mass). There is almost no sign of anisotropy in the effective masses at $\Gamma$. In comparison to the literature on graphene, an LDA study found a small anisotropy in both the valence and conduction band of graphane [58], while an earlier generalised gradient approximation study makes no mention of any such anisotropy [56]

all-valence description is required taking into account the $s, p_{x}, p_{y}$, and $p_{z}$ orbitals of $\mathrm{Si}$, as well as the $\mathrm{H} s$ orbital. This is a direct consequence of the sublattice buckling; an all-valence model is already needed for the description of the silicene lattice (excluding the $\mathrm{H}$ orbitals, naturally) [63].

Table 1.3. Orbital decomposition of the valence and conduction bands of silicane at the $\Gamma$ and $\mathrm{M}$ points according to the local density approximation

\begin{tabular}{lcc}
\hline & $\Gamma$ & $\mathrm{M}$ \\
\hline Silicane val. & $0.23\left(p_{x}^{\mathrm{Si}}+p_{y}^{\mathrm{Si}}\right)$ & $0.05 p_{x}^{\mathrm{Si}}+0.16 p_{y}^{\mathrm{Si}}$ \\
Silicane cond. & $0.09 s^{\mathrm{Si}}+0.05 p_{z}^{\mathrm{Si}}+0.03 s^{\mathrm{H}}$ & $0.07 s^{\mathrm{Si}}+0.01 p_{x}^{\mathrm{Si}}+0.01 p_{y}^{\mathrm{Si}}+0.03 p_{z}^{\mathrm{Si}}$ \\
\hline
\end{tabular}


While a full geometry optimisation of silicane yields an energetically stable configuration, it is necessary to examine its phonon dispersion in order to ascertain whether that configuration is dynamically stable. The phonon dispersion reveals that silicane is stable as there is no sign of any dynamical instability anywhere along the high-symmetry lines of the Brillouin zone (see Fig. 1.5).

The so-called chair-like structure shown in Fig. 1.1 corresponds to the case when $\mathrm{H}$ atoms alternate on the two sides of the sheet such that for each sublattice the $\mathrm{H}$ atom is on a fixed side. In the so-called boat configuration, which is not considered here, the $\mathrm{H}$ atoms alternate in pairs instead, which slightly increases the unit cell size. The latter has been shown to be notably less stable than the chair configuration in the case of graphane [56]; nevertheless the boat configuration of silicane has been found to be stable [52].

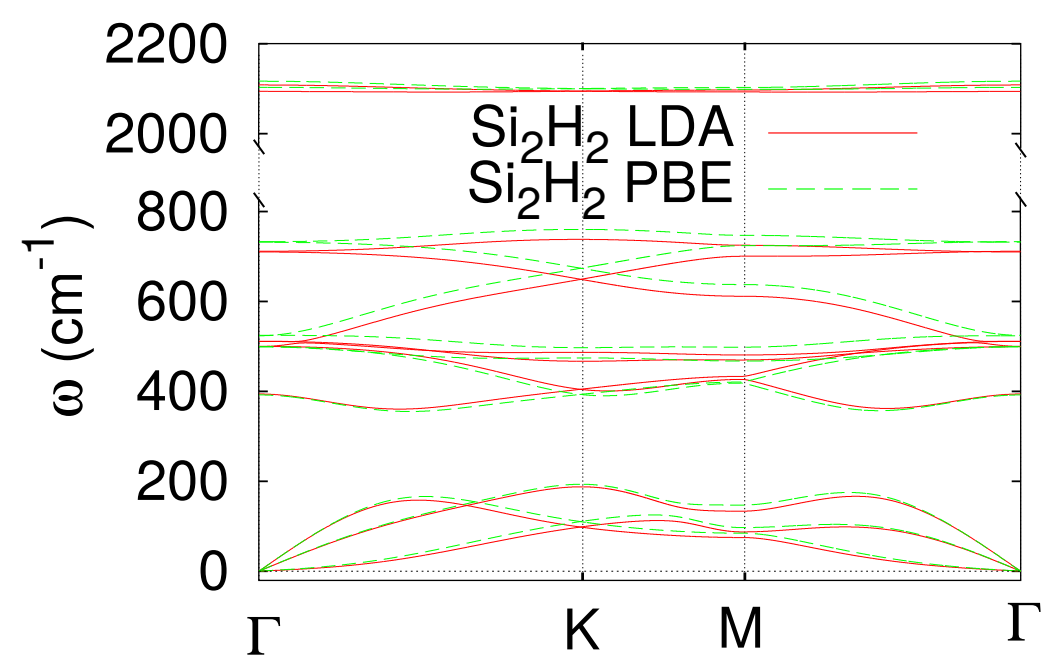

Fig. 1.5. Phonon dispersion of silicane.

\subsection{Tight-binding description of silicene and silicane}

\subsubsection{All-valence tight-binding model of silicene}

The unit cell of silicene comprises two $\mathrm{Si}$ atoms in a honeycomb lattice, and the $A$ and $B$ sublattices of Si atoms exhibit a buckling. The minimum tightbinding model required to describe the band structure in the entire Brillouin zone is an all-valence nearest-neighbour model that takes into account four orbitals per $\mathrm{Si}$ atom (one $s$ and three $p$ orbitals). The tight-binding Hamiltonian is 


$$
H=H_{0}+H_{1},
$$

where

$$
\begin{aligned}
H_{0}= & \sum_{i}\left[\varepsilon_{s} a_{i}^{+} a_{i}+\varepsilon_{p} \sum_{\alpha}\left(b_{i \alpha}^{+} b_{i \alpha}\right)\right] \\
H_{1}= & \gamma_{s s} a_{A}^{+} a_{B}+\sum_{\alpha}\left\{\gamma_{s p} v_{\alpha}^{A B} a_{A}^{+} b_{B \alpha}+\gamma_{p p \sigma}\left(v_{\alpha}^{A B}\right)^{2} b_{A \alpha}^{+} b_{B \alpha}\right. \\
& \left.+\gamma_{p p \pi}\left[1-\left(v_{\alpha}^{A B}\right)^{2}\right] b_{A \alpha}^{+} b_{B \alpha}\right\} \\
& +\sum_{\alpha \neq \beta}\left(\gamma_{p p \sigma} v_{\alpha}^{A B} v_{\beta}^{A B} b_{A \alpha}^{+} b_{B \beta}-\gamma_{p p \pi} v_{\alpha}^{A B} v_{\beta}^{A B} b_{A \alpha}^{+} b_{B \beta}\right)+\text { h.c. }
\end{aligned}
$$

Here, $a^{+}$and $a$ are the creation and annihilation operators of the $s$ electrons of $\mathrm{Si}, b^{+}$and $b$ are the same for the $p$ electrons of Si. In $H_{0}$, parameters $\varepsilon_{s}$ and $\varepsilon_{p}$ are the on-site energies of the $s$ and $p$ orbitals of Si. In $H_{1}, \gamma_{s s}, \gamma_{s p}, \gamma_{p p \sigma}$, and $\gamma_{p p \pi}$ are the nearest-neighbour hoppings between $\mathrm{Si}$ electrons on sublattice $A$ and sublattice $B$. Summations in $i$ go over the $A$ and $B$ sublattices while summations in $\alpha$ and $\beta$ go over $x, y$, and $z \cdot v_{\alpha}^{A B}, v_{\alpha}^{A A^{\prime}}$, and $v_{\alpha}^{A B^{\prime}}$ take into account the orientation of the $p$ orbitals, where $\mathbf{R}$ denotes the coordinates of the atoms. The Hamiltonian in the Slater-Koster approach [59], shown with solid lines separating the $A$ and $B$ sublattice contributions, has the form of an $8 \times 8$ matrix:

$$
\left(\begin{array}{cccc|cccc}
\varepsilon_{s} & & & & H_{s s} & H_{s x} & H_{s y} & H_{s z} \\
& \varepsilon_{p} & & & H_{s x} & H_{x x} & H_{x y} & H_{x z} \\
& & \varepsilon_{p} & & H_{s y} & H_{x y} & H_{y y} & H_{y z} \\
& & \varepsilon_{p} & H_{s z} & H_{x z} & H_{y z} & H_{z z} \\
\hline H_{s s}^{*} & H_{s x}^{*} & H_{s y}^{*} & H_{s z}^{*} & \varepsilon_{s} & & & \\
H_{s x}^{*} & H_{x x}^{*} & H_{x y}^{*} & H_{x z}^{*} & & \varepsilon_{p} & & \\
H_{s y}^{*} & H_{x y}^{*} & H_{y y}^{*} & H_{y z}^{*} & & & \varepsilon_{p} & \\
H_{s z}^{*} & H_{x z}^{*} & H_{y z}^{*} & H_{z z}^{*} & & & & \varepsilon_{p}
\end{array}\right),
$$

where

$$
\begin{aligned}
H_{s s}= & \gamma_{s s} \sum_{B} e^{i \mathbf{k} \cdot\left(\mathbf{R}_{\mathrm{Si}}^{\mathrm{A}}-\mathbf{R}_{\mathrm{Si}}^{\mathrm{Bi}}\right)} \\
H_{s \alpha}= & \gamma_{s p} \sum_{B} v_{\alpha}^{A B} e^{i \mathbf{k} \cdot\left(\mathbf{R}_{\mathrm{Si}}^{\mathbf{A}}-\mathbf{R}_{\mathrm{Si}}^{\mathrm{B}}\right)} \\
H_{\alpha \alpha}= & \gamma_{p p \sigma} \sum_{B}\left(v_{\alpha}^{A B}\right)^{2} e^{i \mathbf{k} \cdot\left(\mathbf{R}_{\mathrm{Si}}^{\mathrm{A}}-\mathbf{R}_{\mathrm{Si}}^{\mathrm{B}}\right)}+\gamma_{p p \pi} \sum_{B}\left[1-\left(v_{\alpha}^{A B}\right)^{2}\right] e^{i \mathbf{k} \cdot\left(\mathbf{R}_{\mathrm{Si}}^{\mathbf{A}}-\mathbf{R}_{\mathrm{Si}}^{\mathrm{B}}\right)} \\
H_{\alpha \beta}= & \gamma_{p p \sigma} \sum_{B} v_{\alpha}^{A B} v_{\beta}^{A B} e^{i \mathbf{k} \cdot\left(\mathbf{R}_{\mathrm{Si}}^{\mathrm{A}}-\mathbf{R}_{\mathrm{Si}}^{\mathrm{B}}\right)} \\
& -\gamma_{p p \pi} \sum_{B} v_{\alpha}^{A B} v_{\beta}^{A B} e^{i \mathbf{k} \cdot\left(\mathbf{R}_{\mathrm{Si}}^{\mathrm{A}}-\mathbf{R}_{\mathrm{Si}}^{\mathrm{B}}\right)}
\end{aligned}
$$


The total number of parameters in this model is six, but one can choose one of the on-site energies to be zero to set the Fermi level, leaving five parameters to fit. The resulting model can be used to provide a simple, semiempirical reproduction of first-principles band structures.

After fitting the model to the HSE06 band structure of silicene, the following values are obtained for the parameters: $\varepsilon_{s}=-6.08 \mathrm{eV}, \gamma_{s s}=-2.55$ $\mathrm{eV}, \gamma_{s p}=-2.74 \mathrm{eV}, \gamma_{p p \sigma}=-2.76 \mathrm{eV}$, and $\gamma_{p p \pi}=-1.28 \mathrm{eV}$.

\subsubsection{All-valence tight-binding model of silicane}

Silicane has a honeycomb lattice as depicted in Fig. 1.1. The unit cell comprises two Si atoms and two $\mathrm{H}$ atoms, and the $A$ and $B$ sublattices of Si atoms exhibit a buckling. The minimum tight-binding model needed to describe the band structure in the entire Brillouin zone is an all-valence second-nearestneighbour model that takes into account four orbitals per Si atom (one $s$ and three $p$ orbitals) and the $s$ orbital of hydrogen. The tight-binding Hamiltonian is

$$
\begin{aligned}
H= & H_{0}+H_{1}+H_{2} \\
H_{0}= & \sum_{i}\left(\varepsilon_{s} a_{i}^{+} a_{i}+\varepsilon_{p} \sum_{\alpha}\left(b_{i \alpha}^{+} b_{i \alpha}\right)+\varepsilon_{s} \mathrm{H} c_{i}^{+} c_{i}\right) \\
H_{1}= & \sum_{i}\left(\gamma_{s^{\mathrm{H}}} c_{i}^{+} a_{i}\right)+\gamma_{s s} a_{A}^{+} a_{B}+\sum_{\alpha}\left(\gamma_{s p} v_{\alpha}^{A B} a_{A}^{+} b_{B \alpha}+\right. \\
& \left.\gamma_{p p \sigma}\left(v_{\alpha}^{A B}\right)^{2} b_{A \alpha}^{+} b_{B \alpha}+\gamma_{p p \pi}\left(1-\left(v_{\alpha}^{A B}\right)^{2}\right) b_{A \alpha}^{+} b_{B \alpha}\right)+ \\
& \sum_{\alpha \neq \beta}\left(\gamma_{p p \sigma} v_{\alpha}^{A B} v_{\beta}^{A B} b_{A \alpha}^{+} b_{B \beta}-\gamma_{p p \pi} v_{\alpha}^{A B} v_{\beta}^{A B} b_{A \alpha}^{+} b_{B \beta}\right)+ \\
& \sum_{\alpha} \gamma_{s^{\mathrm{H}} p} v_{\alpha}^{A B} c_{A}^{+} b_{B \alpha}+h . c . \\
H_{2}= & \sum_{i}\left(\gamma_{s s}^{\prime} a_{i}^{+} a_{i^{\prime}}+\sum_{\alpha}\left(\gamma_{s p}^{\prime} v_{\alpha}^{A A^{\prime}} a_{i}^{+} b_{i^{\prime} \alpha}+\gamma_{p p \sigma}^{\prime}\left(v_{\alpha}^{A A^{\prime}}\right)^{2} b_{A \alpha}^{+} b_{A^{\prime} \alpha}+\right.\right. \\
& \left.\gamma_{p p \pi}^{\prime}\left(1-\left(v_{\alpha}^{A A^{\prime}}\right)^{2}\right) b_{A \alpha}^{+} b_{A^{\prime} \alpha}\right)+ \\
& \sum_{\alpha \neq \beta}\left(\gamma_{p p \sigma}^{\prime} v_{\alpha}^{A A^{\prime}} v_{\beta}^{A A^{\prime}} b_{A \alpha}^{+} b_{A^{\prime} \beta}-\gamma_{p p \pi}^{\prime} v_{\alpha}^{A A^{\prime}} v_{\beta}^{A A^{\prime}} b_{A \alpha}^{+} b_{A^{\prime} \beta}\right)+ \\
& \gamma_{s^{\mathrm{H}} s}^{\prime} c_{A}^{+} a_{B}+\sum_{\alpha} \gamma_{s^{\mathrm{H}} p}^{\prime} v_{\alpha}^{A B^{\prime}} c_{A}^{+} b_{B \alpha}+h . c . \\
v_{\alpha}^{A B}= & \left(\mathbf{R}_{\mathrm{Si}}^{A}-\mathbf{R}_{\mathrm{Si}}^{B}\right)_{\alpha} /\left|\mathbf{R}_{\mathrm{Si}}^{A}-\mathbf{R}_{\mathrm{Si}}^{B}\right| \\
v_{\alpha}^{A A^{\prime}}= & \left(\mathbf{R}_{\mathrm{Si}}^{A}-\mathbf{R}_{\mathrm{Si}}^{A^{\prime}}\right)_{\alpha} /\left|\mathbf{R}_{\mathrm{Si}}^{A}-\mathbf{R}_{\mathrm{Si}}^{A^{\prime}}\right| \\
v_{\alpha}^{A B^{\prime}}= & \left(\mathbf{R}_{\mathrm{Si}}^{A}-\mathbf{R}_{\mathrm{H}}^{B^{\prime}}\right)_{\alpha} / \mid \mathbf{R}_{\mathrm{Si}}^{A}-\mathbf{R}_{\mathrm{H}}^{B^{\prime} \mid}
\end{aligned}
$$

Here, $a^{+}$and $a$ are the creation and annihilation operators of the $s$ electrons of $\mathrm{Si}, b^{+}$and $b$ are the same for the $p$ electrons of $\mathrm{Si}$, while $c^{+}$and $c$ are the same 
for the electrons of the $\mathrm{H}$ atoms. In $H_{0}$, parameters $\varepsilon_{s}$ and $\varepsilon_{p}$ are the on-site energies of the $s$ and $p$ orbitals of $\mathrm{Si}, \varepsilon_{s^{\mathrm{H}}}$ is the on-site energy of the electron of the hydrogen atom. In $H_{1}, \gamma_{s}{ }_{s}$ parameterises the nearest-neighbour hopping between $s$ orbitals of Si and hydrogen, while $\gamma_{s s}, \gamma_{s p}, \gamma_{p p \sigma}$, and $\gamma_{p p \pi}$ are the nearest-neighbour hoppings between Si electrons on sublattices $A$ and $B$. In $H_{2}$, parameters $\gamma_{s s}^{\prime}, \gamma_{s p}^{\prime}, \gamma_{p p \sigma}^{\prime}$, and $\gamma_{p p \pi}^{\prime}$ are the second-nearest-neighbour hoppings between the Si electrons on the same sublattice, while $\gamma_{s^{\mathrm{H}}{ }_{s}}^{\prime}$ and $\gamma_{s}^{\prime} \mathrm{H}_{p}$ are the second-nearest-neighbour hoppings between the orbitals of $\mathrm{Si}$ and hydrogen on different sublattices. Summations in $i$ go over the $A$ and $B$ sublattices while summations in $\alpha$ and $\beta$ go over $x, y, z ; v_{\alpha}^{A B}, v_{\alpha}^{A A^{\prime}}$, and $v_{\alpha}^{A B^{\prime}}$ take into account the orientation of the $p$ orbitals, where $\mathbf{R}$ denotes the coordinates of the atoms. The Hamiltonian in the Slater-Koster approach [59], shown with solid lines separating the $A$ and $B$ sublattice contributions as well as hydrogen contributions, has the form of a $10 \times 10$ matrix:

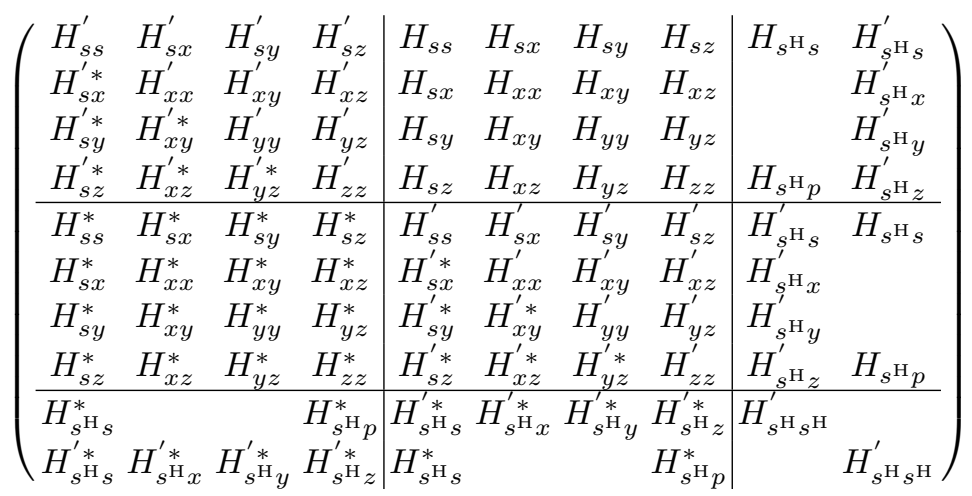

where

$$
\begin{aligned}
& H_{s s}=\gamma_{s s} \sum_{B} e^{i \mathbf{k}\left(\mathbf{R}_{\mathrm{Si}^{\mathrm{i}}}^{\mathrm{A}}-\mathbf{R}_{\mathrm{Si}}^{\mathrm{B}}\right)}, H_{s^{\mathrm{H}} s}=\gamma_{s^{\mathrm{H}} s}, H_{s^{\mathrm{H}} p}=\gamma_{s^{\mathrm{H}} p} \\
& H_{s \alpha}=\gamma_{s p} \sum_{B} v_{\alpha}^{A B} e^{i \mathbf{k}\left(\mathbf{R}_{\mathrm{Si}}^{\mathbf{A}}-\mathbf{R}_{\mathrm{Si}}^{\mathrm{B}}\right)}, H_{s \alpha}^{\prime}=\gamma_{s p}^{\prime} \sum_{A} v_{\alpha}^{A A^{\prime}} e^{i \mathbf{k}\left(\mathbf{R}_{\mathrm{Si}}^{\mathbf{A}}-\mathbf{R}_{\mathrm{Si}}^{\mathrm{A}^{\prime}}\right)} \\
& H_{\alpha \alpha}=\gamma_{p p \sigma} \sum_{B}\left(v_{\alpha}^{A B}\right)^{2} e^{i \mathbf{k}\left(\mathbf{R}_{\mathrm{Si}}^{\mathrm{A}}-\mathbf{R}_{\mathrm{Si}}^{\mathrm{B}}\right)}+\gamma_{p p \pi} \sum_{B}\left(1-\left(v_{\alpha}^{A B}\right)^{2}\right) e^{i \mathbf{k}\left(\mathbf{R}_{\mathrm{Si}}^{\mathrm{A}}-\mathbf{R}_{\mathrm{Si}}^{\mathrm{B}}\right)} \\
& H_{\alpha \beta}=\gamma_{p p \sigma} \sum_{B} v_{\alpha}^{A B} v_{\beta}^{A B} e^{i \mathbf{k}\left(\mathbf{R}_{\mathrm{Si}}^{\mathrm{A}}-\mathbf{R}_{\mathrm{Si}}^{\mathrm{B}}\right)}-\gamma_{p p \pi} \sum_{B} v_{\alpha}^{A B} v_{\beta}^{A B} e^{i \mathbf{k}\left(\mathbf{R}_{\mathrm{Si}}^{\mathrm{A}}-\mathbf{R}_{\mathrm{Si}}^{\mathrm{Bi}}\right)}, \alpha \neq \beta \\
& H_{\alpha \alpha}^{\prime}=\varepsilon_{p}+\gamma_{p p \sigma}^{\prime} \sum_{A}\left(v_{\alpha}^{A A^{\prime}}\right)^{2} e^{i \mathbf{k}\left(\mathbf{R}_{\mathrm{Si}}^{\mathrm{A}}-\mathbf{R}_{\mathrm{Si}}^{\mathrm{A}^{\prime}}\right)}+\gamma_{p p \pi}^{\prime} \sum_{A}\left(1-\left(v_{\alpha}^{A A^{\prime}}\right)^{2}\right) e^{i \mathbf{k}\left(\mathbf{R}_{\mathrm{Si}}^{\mathrm{A}}-\mathbf{R}_{\mathrm{Si}}^{\mathrm{A}^{\prime}}\right)} \\
& H_{\alpha \beta}^{\prime}=\gamma_{p p \sigma}^{\prime} \sum_{A} v_{\alpha}^{A A^{\prime}} v_{\beta}^{A A^{\prime}} e^{i \mathbf{k}\left(\mathbf{R}_{\mathrm{Si}}^{\mathrm{A}}-\mathbf{R}_{\mathrm{Si}}^{\mathrm{A}^{\prime}}\right)}-\gamma_{p p \pi}^{\prime} \sum_{A} v_{\alpha}^{A A^{\prime}} v_{\beta}^{A A^{\prime}} e^{i \mathbf{k}\left(\mathbf{R}_{\mathrm{Si}}^{\mathrm{A}}-\mathbf{R}_{\mathrm{Si}}^{\mathrm{A}^{\prime}}\right)}, \alpha \neq \beta \\
& H_{s^{\mathrm{H}} s}^{\prime}=\gamma_{s^{\mathrm{H}}}^{\prime} \sum_{B} e^{i \mathbf{k}\left(\mathbf{R}_{\mathrm{Si}^{\mathrm{A}}}^{\mathrm{A}}-\mathbf{R}_{\mathrm{H}}^{\mathrm{B}^{\prime}}\right)}, H_{s^{\mathrm{H}^{\mathrm{H}}}}^{\prime}=\varepsilon_{s}^{\mathrm{H}}+\gamma_{s^{\mathrm{H}} s^{\mathrm{H}}}^{\prime} \sum_{A} e^{i \mathbf{k}\left(\mathbf{R}_{\mathrm{Si}^{\mathrm{i}}}^{\mathrm{A}}-\mathbf{R}_{\mathrm{Si}^{\prime}}^{\mathrm{A}^{\prime}}\right)}
\end{aligned}
$$


$H_{s^{\mathrm{H}} \alpha}^{\prime}=\gamma_{s^{\mathrm{H}} p}^{\prime} \sum_{B} v_{\alpha}^{A B^{\prime}} e^{i \mathbf{k}\left(\mathbf{R}_{\mathrm{Si}}^{\mathbf{A}}-\mathbf{R}_{\mathrm{H}} \mathbf{B}^{\mathbf{B}^{\prime}}\right)}, H_{s s}^{\prime}=\varepsilon_{s}+\gamma_{s s}^{\prime} \sum_{A} e^{i \mathbf{k}\left(\mathbf{R}_{\mathrm{Si}}^{\mathbf{A}}-\mathbf{R}_{\mathrm{Si}}^{\mathbf{A}^{\prime}}\right)}$

The total number of parameters in this model is sixteen, but one can choose one of the on-site energies to be zero to set the Fermi level, leaving fifteen parameters to fit. The resulting model can be used to provide a simple semiempirical reproduction of first-principles band structures.

Using the HSE06 band structures as reference, the tight-binding band structure can reproduce the entirety of the DFT valence band and the vicinity of the conduction band at both the $\Gamma$ and $\mathrm{M}$ points (see Fig. 1.6) to within $\approx 1 \mathrm{eV}$ of the band edge. It is important to note here that if secondnearest-neighbour interactions are neglected, the valence band can still be reproduced to within several $\mathrm{eV}$ of the band edge, but the behaviour of the conduction band at the M point cannot, which indicates that the secondnearest-neighbour interactions are responsible for the minimum in the conduction band at the M point. Also, the $d$-shell of Si is likely to affect states in the conduction band. The best fit is achieved with the parameters listed in the legend of Fig. 1.6; the fitting was optimised to give a quantitative description of the valence band and the conduction band near the $\Gamma$ and the M point.

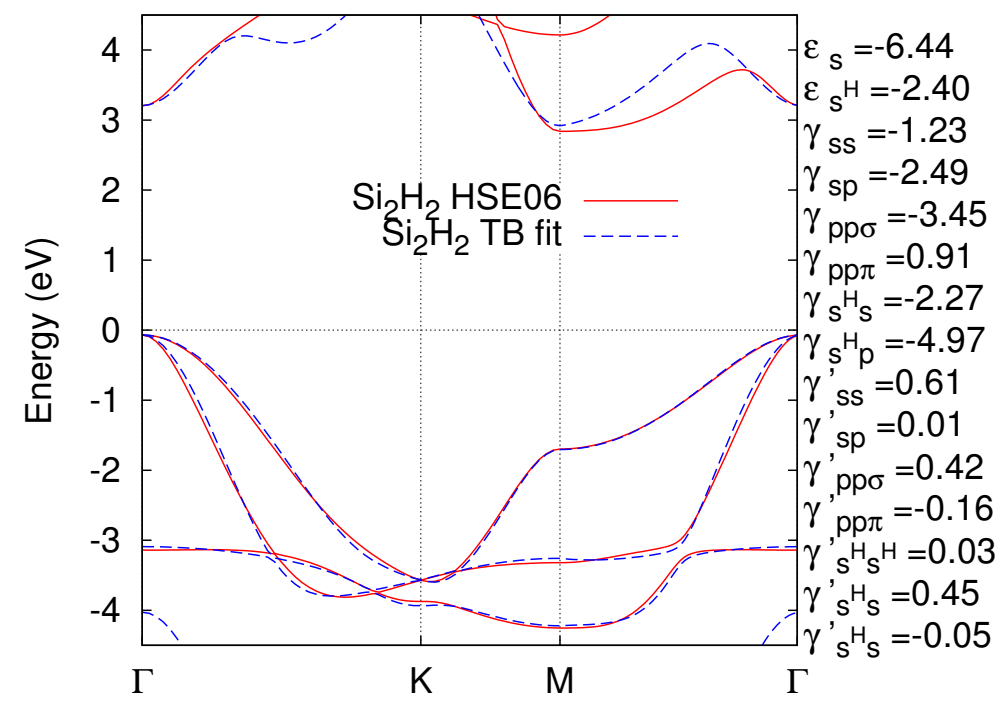

Fig. 1.6. Tight-binding band structure of silicane compared with the HSE06 DFT bands. The parameters of the model are shown in the legend in units of $\mathrm{eV}$. The reference energy level is set by $\varepsilon_{p}=0$ 


\subsection{Silicene in a transverse external electric field}

To exploit the weak buckling of silicene, one can apply an external electric field $E_{z}$ in the $z$ direction, as shown in Fig. 1.7. The main effect of such an electric field is to break the symmetry between the $A$ and $B$ sublattices of silicene's honeycomb structure and hence to open a gap $\Delta$ in the band structure at the hexagonal BZ points $K$ and $K^{\prime}$. In the framework of a simplified nearest-neighbour tight-binding model, which can be thought of as an expansion of the tight-binding model in Sec. 1.3.1 for small wave vectors around the corner of the Brillouin zone, this manifests itself in the form of an energy correction to the on-site energies that is positive for sublattice $A$ and negative for $B$. This difference in on-site energies $\Delta=\mathcal{E}_{A}-\mathcal{E}_{B}$ leads to a spectrum with a gap for electrons in the vicinity of the corners of the BZ: $\mathcal{E}_{ \pm}= \pm \sqrt{(\Delta / 2)^{2}+|v \mathbf{p}|^{2}}$, where $\mathbf{p}$ is the electron "valley" momentum relative to the BZ corner. Opening a gap in graphene by these means would be impossible because the $A$ and $B$ sublattices lie in the same plane.

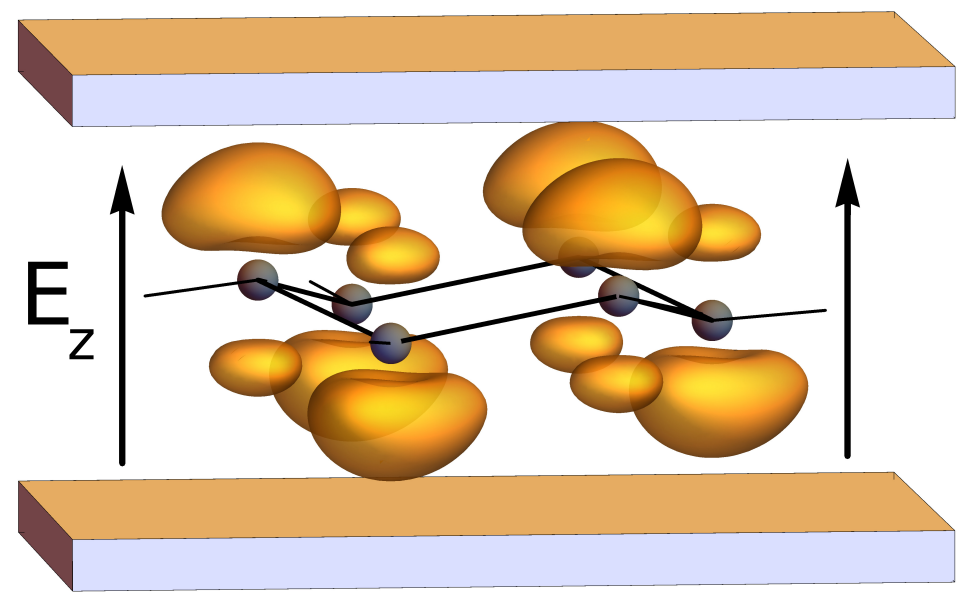

Fig. 1.7. Sketch of silicene in an external electric field, with an illustration of the charge density for the highest occupied valence band in the vicinity of the $\mathrm{K}$ point

A naïve estimate of the electric-field-induced gap in silicene can be made using first-order perturbation theory by diagonalising a $2 \times 2$ Hamiltonian matrix at $\mathbf{p} \rightarrow \mathbf{0}$,

$$
\delta \mathcal{H}\left(E_{z}\right)=e E_{z}\left[\begin{array}{l}
\left\langle\psi_{\mathrm{K}}^{-}|z| \psi_{\mathrm{K}}^{-}\right\rangle\left\langle\psi_{\mathrm{K}}^{-}|z| \psi_{\mathrm{K}}^{+}\right\rangle \\
\left\langle\psi_{\mathrm{K}}^{+}|z| \psi_{\mathrm{K}}^{-}\right\rangle\left\langle\psi_{\mathrm{K}}^{+}|z| \psi_{\mathrm{K}}^{+}\right\rangle
\end{array}\right] .
$$

Here, $\psi_{\mathrm{K}}^{ \pm}$are the degenerate lowest unoccupied and highest occupied KohnSham orbitals at the $\mathrm{K}$ point at $E_{z}=0$, and $z=0$ corresponds to the mid- 
plane of the buckled lattice. This suggests a band gap which opens linearly with the electric field at a rate $d \Delta / d E_{z}=0.554$ and $0.573 \mathrm{e} \AA$ for the wave functions $\psi_{K}$ found using the LDA and PBE functionals, respectively.

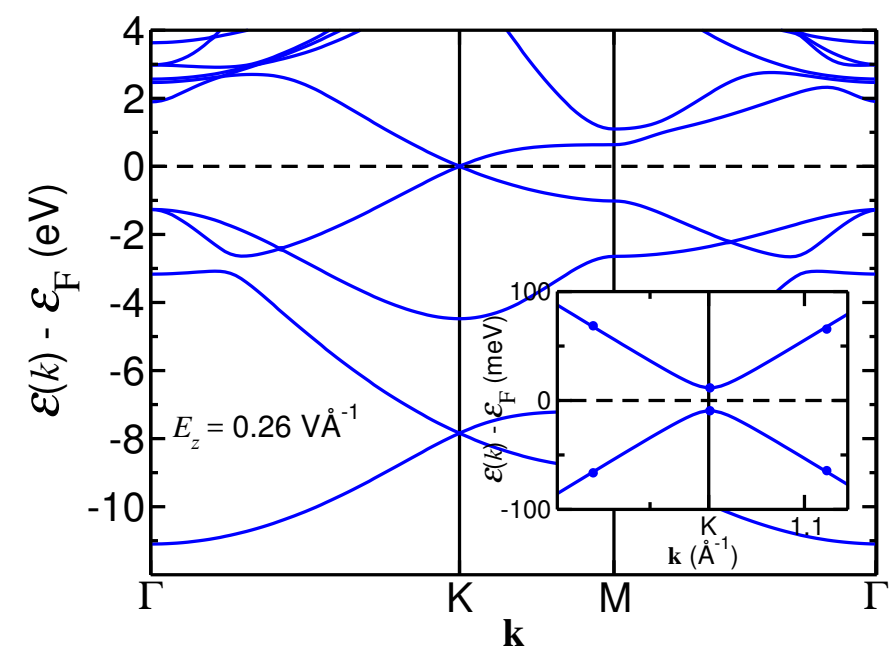

Fig. 1.8. DFT-PBE band structure for silicene in a cell of length $L_{z}=26.5 \AA$ with a plane-wave cutoff energy of $816 \mathrm{eV}$ and a $53 \times 53 \mathrm{k}$-point grid in $E_{z}=0.26$ $\mathrm{V} \AA^{-1}$ external electric field. The zero of the external potential is in the centre of the silicene layer. The dashed line shows the Fermi energy and the inset shows the spectrum near the Fermi level in the vicinity of the $\mathrm{K}$ point

The estimate is in fact only an upper limit for the rate at which the band gap opens, since it neglects screening by the polarisation of the $A$ and $B$ sublattices. In order to obtain an accurate value of the rate at which a band gap can be opened with an electric field, fully self-consistent calculations of the DFT band structure in the presence of an electric field must be carried out. A typical result of such a calculation is shown in Fig. 1.8. At small electric fields, relaxing the structure in the presence of the field does not have a significant effect on the band gap, but the screening of the electric potential by the sublattice polarisation of the electron states makes a substantial difference. The DFT-calculated gaps are gathered in Fig. 1.9. The variation of the band gap $\Delta$ at $\mathrm{K}$ with electric field $E_{z}$ is almost perfectly linear for fields up to $E_{z} \approx 1 \mathrm{~V}^{-1}$. The results for the rate $d \Delta / d E_{z}$ at which a gap is opened are shown in the table inset in Fig. 1.9. The eightfold difference between the self-consistent and the unscreened values of $d \Delta / d E_{z}$ indicates that the system exhibits a strong sublattice polarisability.

Applying a transverse electric field $E_{z}$ in a periodic simulation cell results in a sawtooth potential in the out-of-plane $(z)$ direction. This creates 


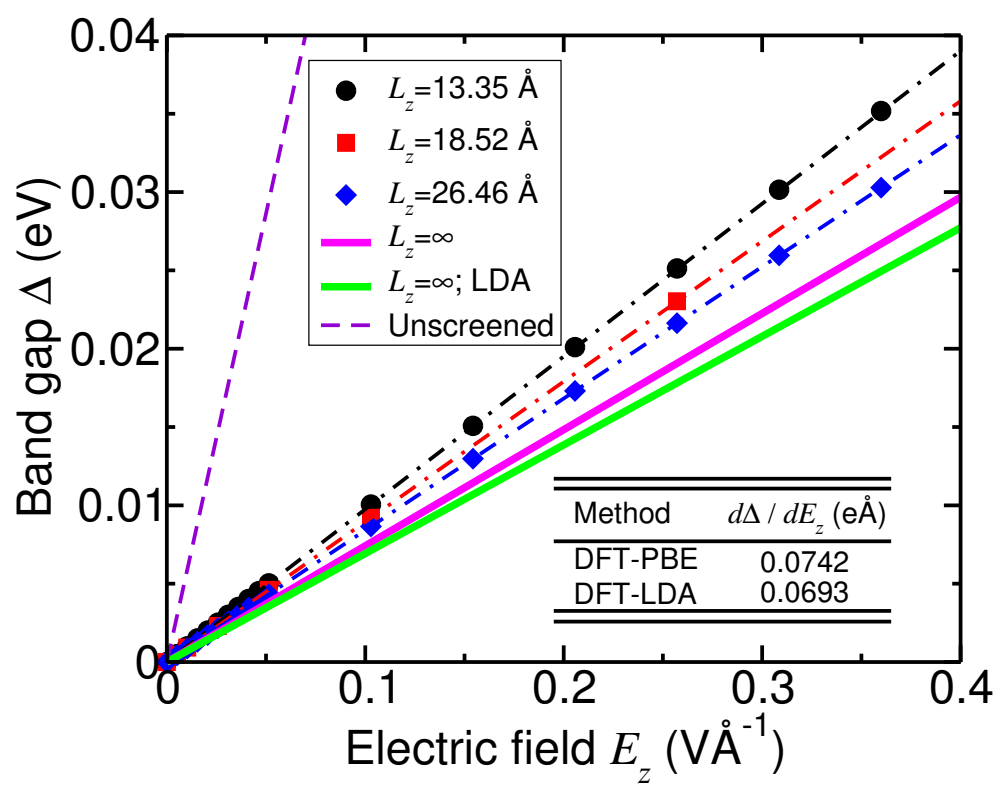

Fig. 1.9. DFT gap against applied electric field $E_{z}$ for silicene with a plane-wave cutoff energy of $816 \mathrm{eV}$ and a $53 \times 53 \mathrm{k}$-point grid. Unless otherwise stated, the PBE functional was used. The box length in the $z$ direction was varied from $L_{z}=13.35$ $\AA$ to $26.46 \AA$. The results have been extrapolated to the limit $L_{z} \rightarrow \infty$ of infinite box length (solid lines). Unscreened band gaps calculated using perturbation theory are also shown. The inset table shows the calculated rate at which the band gap opens

a triangular quantum well in the vacuum region between periodic images of the silicene layer. If a plane-wave basis set is used, the formation of a quasi$2 \mathrm{D}$ electron gas in this spurious quantum well can in principle be described; however, for the cell lengths used and the electric fields considered in Fig. 1.8 , these quantum-well states are unoccupied, as demonstrated by the absence of quadratic bands at $\Gamma$ in the vicinity of the Fermi energy. Hence at low $E_{z}$ the electric-field-induced band gap can be reliably calculated using a plane-wave basis, and a linear dependence of the field-induced gap on $E_{z}$ is obtained. It is found that the gap for a given $E_{z}$ varies with the length of the cell in the $z$ direction; however, the gap can be extrapolated to infinite layer separation using a series of cell lengths that are sufficiently short that no spurious quantum-well states are found close to the Fermi energy.

Several DFT-PBE calculations of the rate at which the gap opens in the presence of a transverse electric field have been reported. Plane-wave basis DFT-PBE calculations yield a rate of 0.0742 eA [4], while localised basis set calculations give values between $0.157 \mathrm{e} \AA[15]$ and $\approx 0.1 \mathrm{e} \AA$ [62], which agree 
to within an order of magnitude. The plane-wave results are illustrated in Fig. 1.8.

It is important to note that the electric field does not simply open a linearly tunable band gap but can also slightly alter the structure, or in extremely large fields it can destabilise it. It was demonstrated [4] by reoptimisation of the geometry in the presence of an electric field that the material is stable in fields up to at least $E_{z} \approx 0.4 \mathrm{~V}^{-1}$.

Figure 1.2 demonstrates what happens to the phonon dispersion in the presence of the electric field. The main effects of a small $E_{z}$ on the phonon dispersion curve are (i) to lift some degeneracies at $\mathrm{K}$ and $\mathrm{M}$ and (ii) to soften one of the acoustic branches, but without making the frequency imaginary. Under much higher electric fields, the honeycomb structure of silicene becomes unstable: $E_{z} \geq 2.6 \mathrm{VA}^{-1}$ causes the lattice parameter to increase without bound when the structure is relaxed. In the phonon calculations, the box length was $L_{z}=19.05 \AA$ and the plane-wave cutoff energy was $435 \mathrm{eV}$. This is slightly different from how the phonons were calculated without the presence of an electric field, because the error due to a finite box length $L_{z}$ is potentially much larger in the presence of a transverse electric field.

\subsection{SO coupling and topological phase transition in silicene}

The effects of SO coupling on the band structures of silicene and silicane yield quite different results. In the case of silicane, which is an indirectgap semiconductor, the effect of SO coupling is limited to splitting some of the bands as shown in the inset in Fig. 1.4. In the case of the semimetal silicene, on the other hand, the effect is much more important, especially when considering the behaviour of the material in an electric field.

\subsubsection{SO induced band gap in silicene}

The PBE band structure of silicene with SO coupling explicitly included in the Hamiltonian is shown in Fig. 1.10. The functional predicts an SO gap of the order of a few $\mathrm{meV}$ at the $\mathrm{K}$ point, while the rest of the band structure barely differs from the nonrelativistic case, in agreement with the LDA functional. The calculated LDA and PBE SO gaps are $1.4 \mathrm{meV}$ and 1.5 $\mathrm{meV}$, respectively, in agreement with the literature [60].

The SO calculations were performed with a plane-wave cutoff of $500 \mathrm{eV}$ and a $24 \times 24 \mathrm{k}$-point grid. The length of the simulation box has negligible influence on the SO gap: the gap is the same with simulation box lengths of $15 \AA$ and $30 \AA$ up to numerical accuracy. 


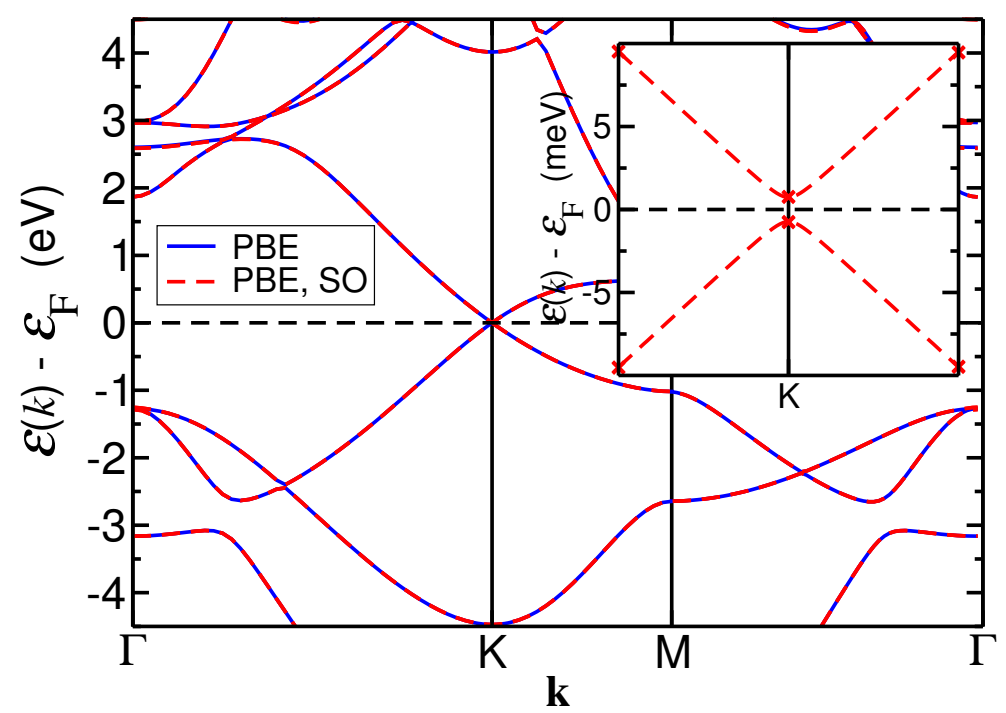

Fig. 1.10. DFT-PBE and DFT-LDA band structures with and without SO coupling taken into account. The inset shows the bands around the K point, revealing a small band gap induced by SO coupling. The width of the bottom panel corresponds to $1 / 200$ of the $\Gamma-\mathrm{K}$ line

\subsubsection{Transition from topological insulator to band insulator state}

In the theory of Dirac electrons on the honeycomb lattice, the SO gap is accounted for by the Kane-Mele term describing, e.g., intrinsic SO coupling in graphene [16]. The Kane-Mele SO coupling and the electric-field induced $A-B$ sublattice asymmetry for electrons in the vicinity of the BZ corners $\mathrm{K}_{ \pm}=( \pm 4 \pi /(3 a), 0)$ in silicene can be incorporated in the Hamiltonian

$$
H_{\mathrm{K}_{ \pm}}=v \mathbf{p} \cdot \sigma+\Delta_{\mathrm{SO}} s_{z} \sigma_{z}+\frac{1}{2} \xi \Delta_{z} \sigma_{z},
$$

where $\xi= \pm 1$ distinguishes between the two valleys, $\mathrm{K}_{+}$and $\mathrm{K}_{-}$, in silicene's spectrum. Here, the Pauli matrices $\sigma_{x}, \sigma_{y}$, and $\sigma_{z}$ act in the space of the electrons' amplitudes on orbitals attributed to the $A$ and $B$ sublattices, $\left(\psi_{A}, \psi_{B}\right)$ for the valley at $\mathrm{K}_{+}$and $\left(\psi_{B},-\psi_{A}\right)$ for the valley at $\mathrm{K}_{-}$. In Eq. (1.10), $s_{z}$ is the electron spin operator normal to the silicene plane, and $\Delta_{\mathrm{SO}}$ and $\Delta_{z}$ are the DFT-calculated SO-coupling and electric-field induced gaps.

The Hamiltonian of Eq. (1.10) generically describes the transition between the $2 \mathrm{D}$ topological and band-gap insulators. Its spectrum,

$$
\begin{aligned}
& \mathcal{E}_{\uparrow \pm}= \pm \sqrt{\frac{1}{4}\left(\Delta_{\mathrm{SO}}+\xi \Delta_{z}\right)^{2}+v^{2} p^{2}}, \\
& \mathcal{E}_{\downarrow \pm}= \pm \sqrt{\frac{1}{4}\left(\Delta_{\mathrm{SO}}-\xi \Delta_{z}\right)^{2}+v^{2} p^{2}},
\end{aligned}
$$


includes two gapped branches, one with a larger gap $\left|\Delta_{\mathrm{SO}}+\Delta_{z}\right|$ and another with a smaller gap $\left|\Delta_{\mathrm{SO}}-\Delta_{z}\right|$. At a critical external electric field $E_{z}^{c} \approx 20$ $\mathrm{mVA} \AA^{-1}, \Delta_{\mathrm{SO}}=\Delta_{z}$, and the smaller gap closes, marking a transition from a topological insulator [16-18] at $\Delta_{\mathrm{SO}}>\Delta_{z}$ to a simple band insulator at $\Delta_{\mathrm{SO}}<\Delta_{z}$. The difference between these two states of silicene is that the topological insulator state supports a gapless spectrum of edge states for the electrons, in contrast to a simple insulator, where the existence of gapless edge states is not protected by topology. However, something similar to the topological properties of Dirac electrons may show up even in the band insulator state of silicene: an interface between two differently gated regions, with electric fields $E_{z}$ and $-E_{z}$ (where $E_{z} \gg E_{z}^{c}$ ), should support a onedimensional gapless band with an almost linear dispersion of electrons [61].

\subsection{Summary}

As illustrated in this chapter, silicene and silicane are prime examples of $2 \mathrm{D}$ materials with rich physics and great application potential. The topological insulator ground state of silicene and its electric field induced transformation into a band insulator with a tunable band gap certainly warrants future study of this fascinating material, while the indirect semiconductor silicane could potentially see good use in silicon-based semiconductor technology.

\section{References}

1. K. S. Novoselov, A. K. Geim, S. V. Morozov, D. Jiang, Y. Zhang, S. V. Dubonos, I. V. Grigorieva, and A. A. Firsov, Science 306, 666 (2004).

2. A. K. Geim and K. S. Novoselov, Nat. Mater. 6, 183 (2007).

3. S. Cahangirov, M. Topsakal, E. Aktürk, H. Şahin, and S. Ciraci, Phys. Rev. Lett. 102, 236804 (2009).

4. N. D. Drummond, V. Zólyomi, and V. I. Fal'ko, Phys. Rev. B 85, 075423 (2012).

5. P. Vogt, P. De Padova, C. Quaresima, J. Avila, E. Frantzeskakis, M. C. Asensio, A. Resta, B. Ealet, and G. Le Lay, Phys. Rev. Lett. 108, 155501 (2012).

6. B. Feng, Z. Ding, S. Meng, Y. Yao, X. He, P. Cheng, L. Chen, and K. Wu, Nano Lett. 12, 3507 (2012).

7. L. Chen, C.-C. Liu, B. Feng, X. He, P. Cheng, Z. Ding, S. Meng, Y. Yao, and K. Wu, Phys. Rev. Lett. 109, 056804 (2012).

8. C. L. Lin, R. Arafune, K. Kawahara, M. Kanno, N. Tsukahara, E. Minamitani, Y. Kim, M. Kawai, and N. Takagi, Phys. Rev. Lett. 110, 076801 (2013).

9. L. Chen, H. Li, B. Feng, Z. Ding, J. Qiu, P. Cheng, K. Wu, and S. Meng, Phys. Rev. Lett. 110, 085504 (2013).

10. P. De Padova, P. Vogt, A. Resta, J. Avila, I. Razado-Colambo, C. Quaresima, C. Ottaviani, B. Olivieri, T. Bruhn, T. Hirahara, T. Shirai, S. Hasegawa, M. C. Asensio, and G. Le Lay, Appl. Phys. Lett. 102, 163106 (2013).

11. A. Resta, T. Leoni, C. Barth, A. Ranguis, C. Becker, T. Bruhn, P. Vogt, and G. Le Lay, Sci. Rep. 3, 2399 (2013). 
12. A. J. Mannix, B. Kiraly, B. L. Fisher, M. C. Hersam, and N. P. Guisinger, ACS Nano 8, 7538 (2014).

13. L. Tao, E. Cinquanta, D. Chiappe, C. Grazianetti, M. Fanciulli, M. Dubey, A. Molle, and D. Akinwande, Nat. Nanotechnol. 10, 227 (2015).

14. P. R. Wallace, Phys. Rev. 71, 622 (1947).

15. Z. Ni, Q. Liu, K. Tang, J. Zheng, J. Zhou, R. Qin, Z. Gao, D. Yu, and J. Lu, Nano Lett. 12, 113 (2012).

16. C. L. Kane and E. J. Mele, Phys. Rev. Lett. 95, 226801 (2005).

17. M. Z. Hasan and C. L. Kane, Rev. Mod. Phys. 82, 3045 (2010).

18. X.-L. Qi and S.-C. Zhang, Rev. Mod. Phys. 83, 1057 (2011).

19. Y. Kubota, K. Watanabe, O. Tsuda, and T. Taniguchi, Science 317, 932 (2007).

20. M. P. Levendorf, C.-J. Kim,L. Brown, P. Y. Huang, R. W. Havener, D. A. Muller, and J. Park, Nature 488, 627 (2012).

21. K. F. Mak, C. Lee, J. Hone, J. Shan, and T. F. Heinz, Phys. Rev. Lett. 105, 136805 (2010).

22. B. Radisavljevic, A. Radenovic, J. Brivio, V. Giacometti, and A. Kis, Nat. Nanotechnol. 6, 147 (2011).

23. B. Radisavljevic, M. B. Whitwick, and A. Kis, ACS Nano 5, 9934 (2011).

24. T. Georgiou, R. Jalil, B. D. Belle, L. Britnell, R. V. Gorbachev, S. V. Morozov, Y.-J. Kim, A. Gholinia, S. J. Haigh, O. Makarovsky, L. Eaves, L. A. Ponomarenko, A. K. Geim, K. S. Novoselov, and A. Mishchenko, Nat. Nanotechnol. 8, $100(2013)$.

25. J. N. Coleman, M. Lotya, A. O'Neill, S. D. Bergin, P. J. King, U. Khan, K. Young, A. Gaucher, S. De, R. J. Smith, I. V. Shvets, S. K. Arora, G. Stanton, H.-Y. Kim, K. Lee, G. T. Kim, G. S. Duesberg, T. Hallam, J. J. Boland, J. J. Wang, J. F. Donegan, J C. Grunlan, G. Moriarty, A. Shmeliov, R. J. Nicholls, J. M. Perkins, E. M. Grieveson, K. Theuwissen, D. W. McComb, P. D. Nellist, and V. Nicolosi, Science 331, 568 (2011).

26. C. Ataca, H. Sahin, and S. Ciraci, J. Phys. Chem. C 116, 8983 (2012).

27. D. Braga, L. I. Gutiérrez, H. Berger, and A. F. Morpurgo, Nano Lett. 12, 5218 (2012).

28. A. Kormányos, V. Zólyomi, N. D. Drummond, P. Rakyta, G. Burkard, and V. I. Fal'ko, Phys. Rev. B 88, 045416 (2013).

29. A. Kormányos, V. Zólyomi, N. D. Drummond, and G. Burkard, Phys. Rev. X 4, 011034 (2014).

30. A. Kormányos, G. Burkard, M. Gmitra, J. Fabian, V. Zólyomi, N. D. Drummond, and V. I. Fal'ko, 2D Materials 2, 022001 (2015).

31. D. MacNeill, C. Heikes, K. F. Mak, Z. Anderson, A. Kormányos, V. Zolyomi, J. Park, and D. C. Ralph, Phys. Rev. Lett. 114, 037401 (2015).

32. V. Zólyomi, N. D. Drummond, and V. I. Fal'ko, Phys. Rev. B 87, 195403 (2013).

33. V. Zólyomi, N. D. Drummond, and V. I. Fal'ko, Phys. Rev. B 89, 205416 (2014).

34. F. Liu, H. Shimotani, H. Shang, T. Kanagasekaran, V. Zólyomi, N. Drummond, V. I. Fal'ko, and K. Tanigaki, ACS Nano 8, 752 (2014).

35. G. W. Mudd, A. Patanè, Z. R. Kudrynskyi, M. W. Fay, O. Makarovsky, L. Eaves, Z. D. Kovalyuk, V. Zólyomi, V. Falko, Appl. Phys. Lett. 105, 221909 (2014).

36. D. C. Elias, R. R. Nair, T. M. G. Mohiuddin, S. V. Morozov, P. Blake, M. P. Halsall, A. C. Ferrari, D. W. Boukhvalov, M. I. Katsnelson, A. K. Geim, and K. S. Novoselov, Science 323, 610 (2009). 
37. E. Bianco, S. Butler, S. Jiang, O. D. Restrepo, W. Windl, and J. E. Goldberger, ACS Nano 7, 4414 (2013).

38. Z. Zólyomi, J. R. Wallbank, and V. I. Fal'ko, 2D Materials 1, 011005 (2014).

39. S. J. Clark, M. D. Segall, C. J. Pickard, P. J. Hasnip, M. I. J. Probert, K. Refson, and M. C. Payne, Z. Kristallogr. 220, 567 (2005).

40. K. Refson, P. R. Tulip, and S. J. Clark, Phys. Rev. B 73, 155114 (2006).

41. G. Kresse and J. Furthmüller, Phys. Rev. B 54, 11169 (1996).

42. J. P. Perdew, K. Burke, and M. Ernzerhof, Phys. Rev. Lett. 77, 3865 (1996).

43. J. Heyd, G. E. Scuseria, and M. Ernzerhof, J. Chem. Phys. 118, 8207 (2003).

44. A. V. Krukau, O. A. Vydrov, A. F. Izmaylov, and G. E. Scuseria, J. Chem. Phys. 125, 224106 (2006).

45. G. P. Francis and M. C. Payne, J. Phys.: Condens. Matter 2, 4395 (1990).

46. R. Winkler and U. Zülicke, Phys. Rev. B 82, 245313 (2010).

47. L. Pan, H. J. Liu, Y. W. Wen, X. J. Tan, H. Y. Lv, J. Shi, and X. F. Tang, Phys. Lett. A 375, 614 (2011).

48. M. Houssa, A. Dimoulas, and A. Molle, J. Phys.: Condens. Matter 27, 253002 (2015).

49. P. E. Blöchl, Phys. Rev. B 50, 17953 (1994).

50. F. Favot and A. D. Corso, Phys. Rev. B 60, 11427 (1999).

51. L. C. Lew Yan Voon, E. Sandberg, R. S. Aga, and A. A. Farajian, Appl. Phys. Lett. 97, 163114 (2010).

52. M. Houssa, E. Scalise, K. Sankaran, G. Pourtois, V. V. Afanas'ev, and A. Stesmans, Appl. Phys. Lett. 98, 223107 (2011).

53. J. C. Garcia, D. B. de Lima, L. V. C. Assali, and J. F. Justo, J. Phys. Chem. C 115, 13242 (2011).

54. O. Pulci, P. Gori, M. Marsili, V. Garbuio, R. Del Sole, and F. Bechstedt, Eur. Phys. Lett. 98, 37004 (2012).

55. W. Wei and T. Jacob, Phys. Rev. B 88, 045203 (2013).

56. J. O. Sofo, A. S. Chaudhari, and G. D. Barber, Phys. Rev. B 75, 153401 (2007).

57. S. Park, B. Lee, S. H. Jeon, and S. Han, Curr. Appl. Phys. 11, S337 (2011).

58. L. Liu and Z. Shen, Appl. Phys. Lett. 95, 252104 (2009).

59. J. C. Slater and G. F. Koster, Phys. Rev. 94, 1498 (1954).

60. C.-C. Liu, W. Feng, and Y. Yao, Phys. Rev. Lett. 107, 076802 (2011).

61. G. W. Semenoff, V. Semenoff, and F. Zhou, Phys. Rev. Lett. 101, 087204 (2008).

62. H. Hakan Gürel, V. Ongun Özçelik, and S. Ciraci, J. Phys. Condens. Matter 25, 305007 (2013).

63. Cheng-Cheng Liu, Hua Jiang, and Yugui Yao, Phys. Rev. B 84, 195430 (2011). 